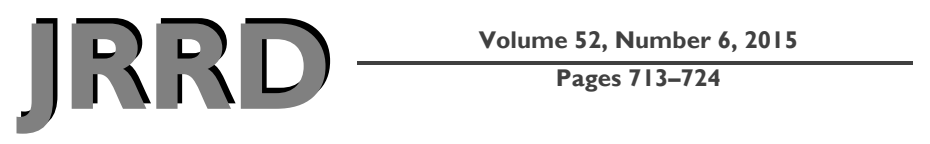

\title{
Core muscle characteristics during walking of patients with multiple sclerosis
}

\author{
Nathaniel B. Ketelhut, BS; ${ }^{1}$ John H. Kindred, MS; ${ }^{1}$ Mark M. Manago, MS; ${ }^{2}$ Jeffrey R. Hebert, PhD; $^{2}$ Thorsten \\ Rudroff, $\mathbf{P h D}^{\mathbf{1}^{*}}$ \\ ${ }^{1}$ Department of Health and Exercise Science, Colorado State University, Fort Collins, CO; ${ }^{2}$ School of Medicine, Uni- \\ versity of Colorado Denver, Denver, CO
}

\begin{abstract}
The purpose of this study was to investigate core muscle characteristics during walking in patients with multiple sclerosis (MS). Eight patients (4 men) with relapsing-remitting MS (aged 44.9 +/- $8.6 \mathrm{yr}$ ) and sex-matched controls (37.9 +/$8.4 \mathrm{yr}$ ) walked on a treadmill for $15 \mathrm{~min}$ at a self-selected speed. Positron emission tomography/computed tomography imaging was used to measure core muscle activity immediately after walking based on glucose uptake. Activity was not different between the MS and control group for any of the identified muscles $(p>0.28)$. Within the MS group, side differences in activity were identified in the lateral flexor group, the external and internal obliques, and the rectus abdominis $(p<0.05)$, with the lessaffected side being activated more. Furthermore, greater muscle volume was found on the more-affected side of the transversus abdominis, quadratus lumborum, and the low-back extensor group $(p<0.03)$. These muscle characteristics suggest patients with MS utilize compensatory mechanisms during walking to maintain balance and posture. These strategies likely result in increased muscle energy cost and early fatigability.
\end{abstract}

Key words: autoimmune disease, balance, computed tomography, disability, glucose uptake heterogeneity, muscle activation, muscle activity, muscle volume, positron emission tomography, stability, trunk.

\section{INTRODUCTION}

Multiple sclerosis (MS) is an inflammatory autoimmune disease of the central nervous system that affects up to 2 million people worldwide [1]. Lower-limb muscle weakness [2], with greater weakness on one side of the body [3-4], is a common symptom and may contribute to the walking difficulties frequently experienced by patients with MS [5]. Moreover, patients with MS have been shown to exhibit decreased postural and trunk control [67], although very little is known about core muscle activity during walking in this population.

Traditionally, muscle activity is measured using electromyography (EMG). This technique provides high temporal resolution of surface muscle activity but is limited in its ability to evaluate deep muscles such as the quadratus

Abbreviations: CMSC $=$ Consortium of Multiple Sclerosis Centers, $\mathrm{CT}=$ computed tomography, $\mathrm{EMG}=$ electromyography, EO $=$ external oblique, $\mathrm{FDG}=\left[{ }^{18} \mathrm{~F}\right]$-Fluorodeoxyglucose, $\mathrm{GUh}=$ glucose uptake heterogeneity, $\mathrm{IO}=$ internal oblique, LB Ext $=$ low-back extensor group, MASS $=$ Modified Ashworth spasticity scale, $\mathrm{MS}=$ multiple sclerosis, NARCOMS $=$ North American Research Committee on Multiple Sclerosis, PDDS = Patient Determined Disease Steps, PET = positron emission tomography, $\mathrm{QL}=$ quadratus lumborum, $\mathrm{RA}=$ rectus abdominis, $\mathrm{ROI}=$ region of interest, $\mathrm{SD}=$ standard deviation, $\mathrm{sEMG}=$ surface electromyography, SUV = standardized uptake value, $\mathrm{TA}=$ transversus abdominis.

*Address all correspondence to Thorsten Rudroff, PhD; 201 D Moby B Complex, Fort Collins, CO 80523; 970-4918655. Email: Thorsten.Rudroff@colostate.edu http://dx.doi.org/10.1682/JRRD.2015.01.0006 
lumborum (QL) and transversus abdominus (TA). Furthermore, data from surface EMG (sEMG) can be compromised due to interference or "crosstalk" from other muscles, and the equipment can experience environmental interference during dynamic tasks. Intramuscular EMG has limitations as well, most notably its invasive nature and that it only measures the activity of a very small portion of the muscle.

Positron emission tomography (PET)/computed tomography $(\mathrm{CT})$ imaging, with the glucose analog $\left[{ }^{18} \mathrm{~F}\right]$ Fluorodeoxyglucose (FDG), can calculate cumulative muscle activity and skeletal muscle properties such as volume without the limitations commonly experienced with EMG. Previous studies have used FDG-PET/CT to measure entire muscle activity, muscle fiber activity, and muscle volume $[4,8-12]$. Muscle activity is quantified by the standardized uptake value (SUV), which directly represents the cumulative muscle activity of the performed task. Muscle fiber activity, or glucose uptake heterogeneity (GUh), is measured by the spatial distribution of FDG within the muscle [1012]. Furthermore, muscle volume can be measured from $C T$ imaging that is often performed just prior to PET imaging [10,12-13].

The activation of the core musculature has been suggested to serve a variety of purposes during walking. In nondisabled subjects, sEMG has been used to show that several core muscles are active throughout the gait cycle and that several, including the rectus abdominis (RA), external oblique (EO), and internal oblique (IO), can have increased activation during specific phases, such as midstance or foot-strike [14-15].

Swinnen et al. used sEMG to investigate the activity of the core muscles during treadmill walking with and without body weight support in patients with MS [15]. The authors found greater core muscle activation on one side of the body with increasing levels of body weight support than with no support. However, more- and lessaffected sides of the patients were not defined, so it was difficult to determine whether weaker or stronger sides of the core were being activated. Furthermore, it is not clear which muscles are most active while walking with no body weight support.

Despite the general lack of understanding of the importance of the core musculature to walking, core muscles have been targeted for intervention to improve gait and postural instability in patients with MS [16-18]. A further understanding of core muscle activity during walking is needed.
Altered muscle activation strategies could lead to increased performance fatigability and contribute to the impaired balance and postural stability frequently experienced by patients with MS. Therefore, the purpose of this study was to investigate the characteristics of the core musculature in patients with MS and nondisabled controls while walking at a self-selected speed using the innovative approach of FDG-PET/CT.

\section{METHODS}

Eight mildly disabled patients with relapsing-remitting MS (4 men) and eight sex-matched nondisabled controls participated in the study. Recruitment was completed through the Rocky Mountain MS Center and University of Colorado Anschutz Medical Campus study announcement letter. Participants were screened via telephone interview to determine eligibility. Requirements to participate in the study for patients with MS included a confirmed diagnosis of MS, age between 18 and $55 \mathrm{yr}$, being able to walk 15 min without assistance, minimal spasticity $(<2$ for the legs on the Modified Ashworth spasticity scale [MASS]), and no change in disease progression within the last 3 mo. Exclusion criteria for patients with MS included any medical diagnosis with contraindications to exercise, relapse within the last $3 \mathrm{mo}$, unrelated condition that would exacerbate fatigue, and history of seizures. The eight controls were without neurological, muscular, or skeletal disease. All participants signed informed consent approved by the Colorado Multiple Institutional Review Board and were in accordance with the Declaration of Helsinki.

\section{Experimental Protocol}

All experiments were performed during the morning hours at the Colorado Translational Research Imaging Center. Patients with MS were assessed for disability levels using the Patient Determined Disease Steps (PDDS), which has been validated and shows a high correlation to the Expanded Disability Status Scale [19-23]. Measurements of height, weight, leg spasticity (using the MASS), comfortable walking speed, and muscle strength preceded 15 min of treadmill walking. The more- and lessaffected sides of patients with MS and strong/weak sides of nondisabled controls were determined from cumulative leg strength since it is known that leg muscle groups are most commonly affected in patients with MS [6,2426]. The maximal voluntary contraction forces for knee 
extension and flexion of both legs were summed for each participant, and the leg with the highest additive forces was classified as the stronger/less-affected leg. A full description of the leg strength methodology can be found in Rudroff et al. [4]. Furthermore, there is currently no accepted method to assess side differences in maximal strength in the core [27], which makes the legs a logical site to assess the more- and less-affected sides of this population.

\section{Walking Protocol}

Following the leg strength testing, participants' plasma glucose levels were tested via finger stick to ensure that the glucose uptake measurement began from comparable baseline conditions. A 22-gauge intravenous catheter was then placed into an antecubital vein in the subject's right arm for injection of FDG. Subjects then walked on a treadmill (Performance $400 \mathrm{C}$, ProForm; Logan, Utah) for $15 \mathrm{~min}$ at a comfortable, predetermined self-selected speed, and any adjustments to speed were made within the first $2 \mathrm{~min}$. Two min after the start of walking, $\approx 8$ millicurie of FDG in $10 \mathrm{~mL}$ of saline was infused into the vein via the inserted catheter. Immediately following walking, the catheter was removed and subjects were guided into the PET/CT camera.

\section{Positron Emission Tomography/Computed Tomography Imaging and Analysis}

Imaging was performed with a Philips Hybrid Gemini TF 64 scanner (Philips Healthcare; Cleveland, Ohio). CT imaging was performed first and immediately followed by PET imaging with the subject's body position secured to maintain coregistration. Imaging was performed using a standard Colorado Translational Research Imaging Center protocol, as done previously by our laboratory $[4,12]$.

Image analysis was completed using Analyze 11.0 (Analyze Direct; Rochester, Minnesota). Muscles were first identified on CT images, and semiautomatic thresholds were set for bone, fat, and muscle tissue. Muscle tissue was classified with a Hounsfield unit range of 0 to 150 [28-29], which ensured the exclusion of intramuscular fat. One investigator drew regions of interest (ROIs) for the core muscles by circling the muscle on each transaxial slice that it could be identified on. Individual muscles were classified by function: (1) trunk flexor: RA; (2) trunk extensors: QL and low-back extensor group (LB Ext) including the multifidus, longissimus, and iliocostalis;
(3) lateral flexors: EO, IO, and TA. The LB Ext were identified inferior to the 11th thoracic vertebrae to match the level of the RA observed in the CT images. ROI object maps were applied to the participants' PET image for quantification of the mean SUV.

SUV $=$ [average tissue radioactivity concentration corrected for time / (injected dose / subject body mass)]

The spatial distribution of fiber activity within each muscle was assessed using GUh. GUh of each ROI was calculated using the following equation: $\mathrm{GUh}=$ (standard deviation [SD] $\left./ \mathrm{SUV}_{\text {mean }}\right) \times 100$ [10-11]. The volume of each ROI was calculated from the CT images using Analyze 11.0 and converted from millimeters cubed to centimeters cubed for analysis.

\section{Statistical Analysis}

A Student $t$-test was used to compare subject characteristics between groups. A separate repeated-measures analysis was performed for each muscle. The withinsubjects factor was side and the between-subjects factor was condition (MS or Control). A side $\times$ condition interaction term was also included in the model, and a random effect for subject (nested within condition) was used to account for repeated measures. Contrasts were used to estimate and test comparisons of interest. Given the interest in differences between sides in patients with MS, within-group comparisons of side were performed. Where appropriate, a Tukey adjustment was used to control for multiple testing (separately for each response variable).

A separate repeated-measures analysis was performed for each variable (SUV, volume, and GUh). The within-subjects factor was muscle (EO, IO, TA, QL, and LB Ext) and the between-subjects factor was condition (MS or Control). A muscle $\times$ condition interaction term was also included in the model, and a random effect for subject (nested within condition) was used to account for repeated measures. Contrasts were used to estimate and test comparisons of interest. A Tukey adjustment was used to control for multiple testing (separately for each response variable).

All statistical analysis was performed using SAS 9.4 (SAS Institute Inc; Cary, North Carolina) with the significance set at a level of $\alpha<0.05$. Data are reported as mean \pm $\mathrm{SD}$ in the text and mean \pm standard error in figures. 


\section{RESULTS}

Eight patients with MS and eight nondisabled controls participated in this study. However, the PET image of one control participant did not reconstruct properly and was excluded from the SUV and GUh analysis. Therefore, seven nondisabled controls were used in the SUV and GUh analysis, and eight controls were used in the muscle volume analysis. The MS and control groups did not differ in age $(44.9 \pm 8.6 \mathrm{yr}, 37.9 \pm 8.4 \mathrm{yr}, p=0.12)$, height $(171 \pm 8 \mathrm{~cm}$, $176 \pm 7 \mathrm{~cm}, p=0.95)$, or weight $(78.2 \pm 3.3 \mathrm{~kg}, 78.2 \pm$ $6.3 \mathrm{~kg}, p=0.98)$. Patients with MS walked at a slower self-selected speed than the nondisabled controls (1.11 \pm $0.22 \mathrm{~m} / \mathrm{s}, 1.37 \pm 0.13 \mathrm{~m} / \mathrm{s}, p=0.01)$. Furthermore, patients with MS were classified as having low levels of disability determined from their PDDS scores (median $=2$, range $=$ $0-4$ ) and their MASS scores (median $=1$, range $=0-1^{+}$) [4]. Plasma glucose concentration immediately prior to the infusion of the FDG was similar for the patients with MS and nondisabled controls $(84 \pm 6 \mathrm{mg} / \mathrm{dL}, 79 \pm 8 \mathrm{mg} / \mathrm{dL}, p=$ 0.2 ). As previously reported by Kindred et al. [12], the magnitude of difference in strength between the legs of patients with MS was greater than that of nondisabled controls. Furthermore, the patients with MS had no visibly noticeable gait impairments while walking. "More/lessaffected" refers to the weak and strong sides, respectively, of the MS group; "strong/weak" refers to the control group.

\section{Muscle Activity}

One outlier was identified as being greater than $2 \mathrm{SD}$ above the mean of the less-affected QL of the MS group, and this value was replaced by the less-affected QL group mean (calculated with the outlying value removed). As indicated by the representative image, patients with MS showed side differences in SUV of core muscles (Figure 1). There were no main effects or interactions for side or condition for any of the muscles $(p>0.05)$. However, within-group comparisons between sides revealed that the lateral flexor muscle group on the less-affected side had greater activity than the more-affected side in the MS group $(p=0.03)$ (Figure 2). Specifically, the less-affected side had greater activity within the EO, IO, and RA $(p<0.05)$ (Figure 3(a)). Within the control group, no differences were seen between the strong and weak sides of the lateral flexor muscle group $(p=$ $0.89)$ or individual muscles $(p>0.45)$.

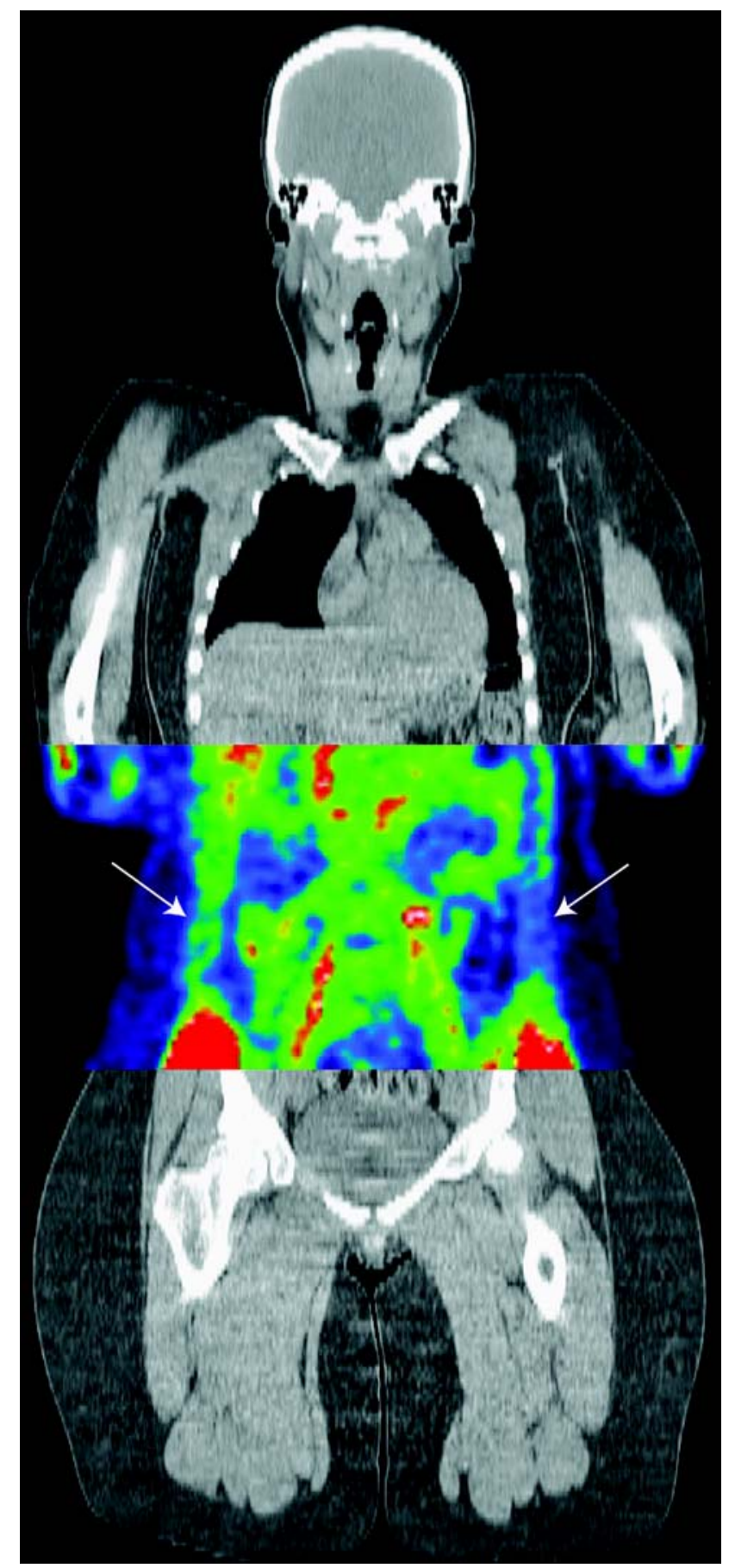

Figure 1.

Positron emission tomography and computed tomography images of patient with multiple sclerosis showing imbalanced muscle activation of lateral flexors. Arrows point to increased activation as indicated by greater $\left[{ }^{18} \mathrm{~F}\right]$-Fluorodeoxyglucose uptake on less-affected side and decreased activity on moreaffected side. Red denotes greatest glucose uptake, while black denotes least. 


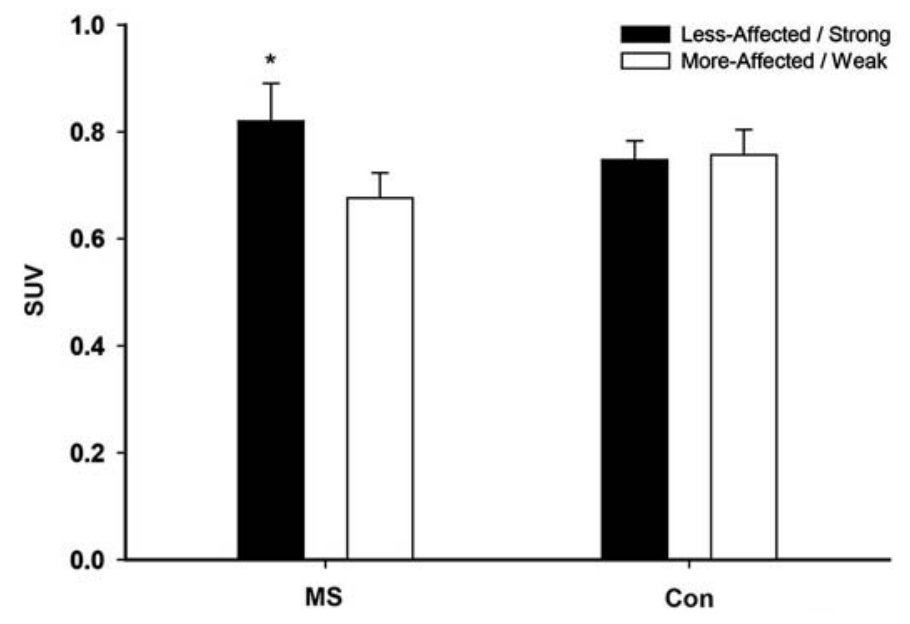

Figure 2.

Mean standardized uptake value (SUV) of lateral flexor group in patients with multiple sclerosis (MS) and nondisabled controls (Con). ${ }^{*} p<0.05$ between more- and less-affected sides.

There was no main effect for condition $(p=0.79)$, and no muscle $\times$ condition interaction was found $(p=$ $0.28)$. However, a main effect for muscle was found ( $p<$ $0.01)$. Post hoc analyses showed differences between the EO and QL/TA/LB Ext $(p<0.01)$. Within-group comparisons of muscle revealed that only the EO and LB Ext muscle groups differed in activity in the MS group, with the LB Ext group being activated more $(p=0.02)$ (Figure 3(b)). The TA and QL each had greater activity than the EO in the control group $(p<0.04)$.

\section{Muscle Volume}

For the majority of muscles, there were no significant main effects or interactions $(p>0.07)$. The single exception was a significant main effect for side in the QL $(p=$ $0.04)$. Within-group comparisons of side showed no difference in volume of the lateral flexor muscle group between the less/more-affected sides in the patients with MS or the strong/weak sides of the controls $(p>0.44)$. However, the less-affected TA, QL, and LB Ext had a lower volume within the MS group $(p<0.03)$ (Figure 4(a)). No differences were found between the strong and weak sides of the individual muscles within the control group $(p>0.16)$.

There was no main effect for condition or muscle $\times$ condition interaction $(p>0.11)$, but a main effect for muscle was identified $(p<0.01)$. Post hoc analyses showed differences between the EO and LB Ext/RA/TA/ QL, IO and LB Ext/RA/TA/QL, LB Ext and QL/RA/TA,
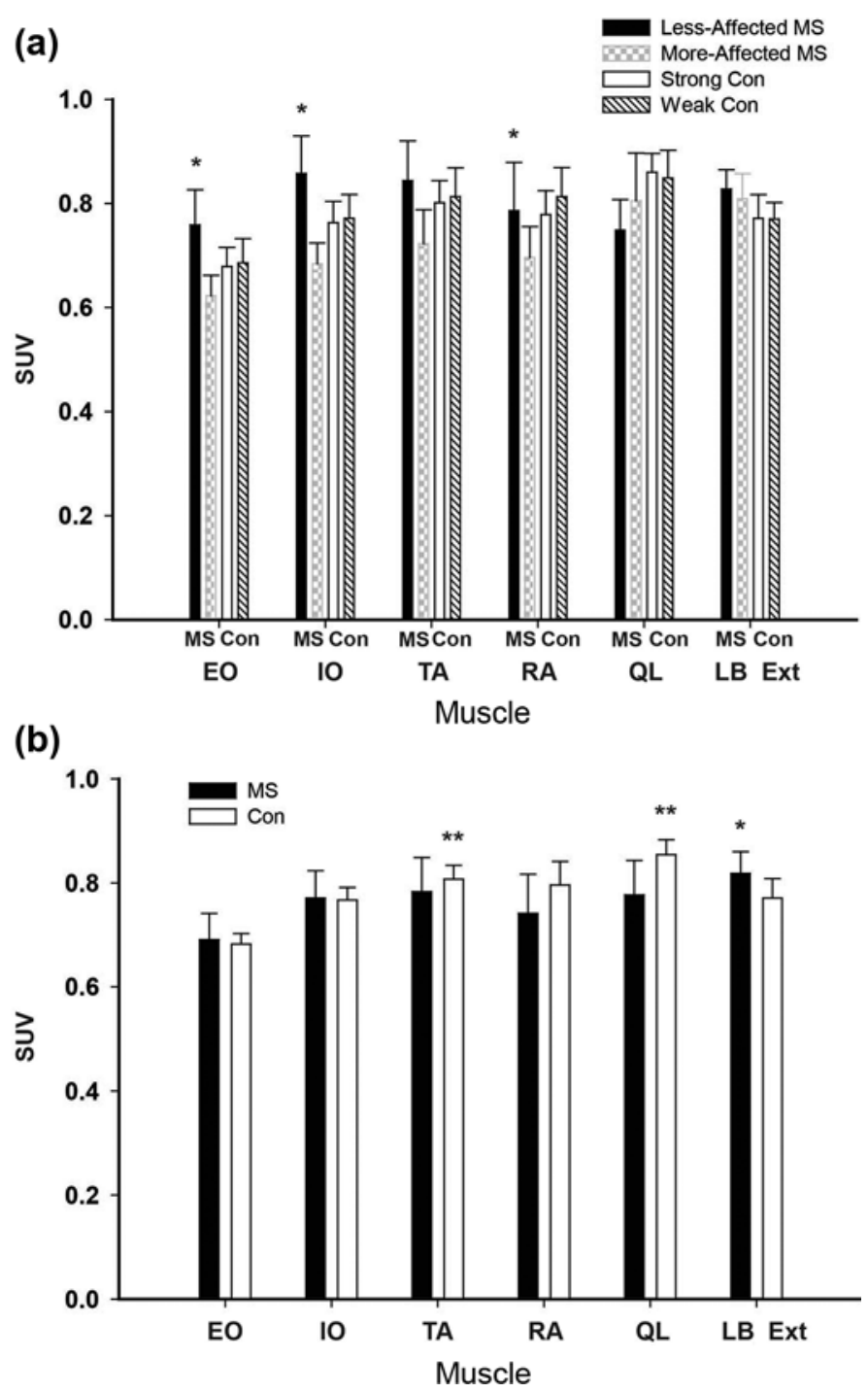

Figure 3.

(a) Mean standardized uptake value (SUV) in patients with multiple sclerosis (MS) and nondisabled controls (Con) between sides of specific muscles and muscle groups. ${ }^{*} p<0.05$ between more and less-affected sides. (b) No difference in mean SUV between specific muscles or muscle groups of patients with MS and nondisabled controls. "Greater SUV than EO in patients with MS $(p<0.05)$. ${ }^{*}$ Greater SUV than EO in nondisabled controls $(p<0.05)$. EO = external oblique, $\mathrm{IO}=$ internal oblique, LB Ext = low-back extensor group (multifidus, longissimus, iliocostalis), $\mathrm{QL}=$ quadratus lumborum, $\mathrm{RA}=$ rectus abdominis, $\mathrm{TA}=$ transversus abdominis.

RA and TA, and QL and RA $(p<0.04)$. Furthermore, between-group comparisons of muscle showed that patients with MS had lower volume in the LB Ext muscle group than nondisabled controls $(p=0.01)$ (Figure 4(b)). 

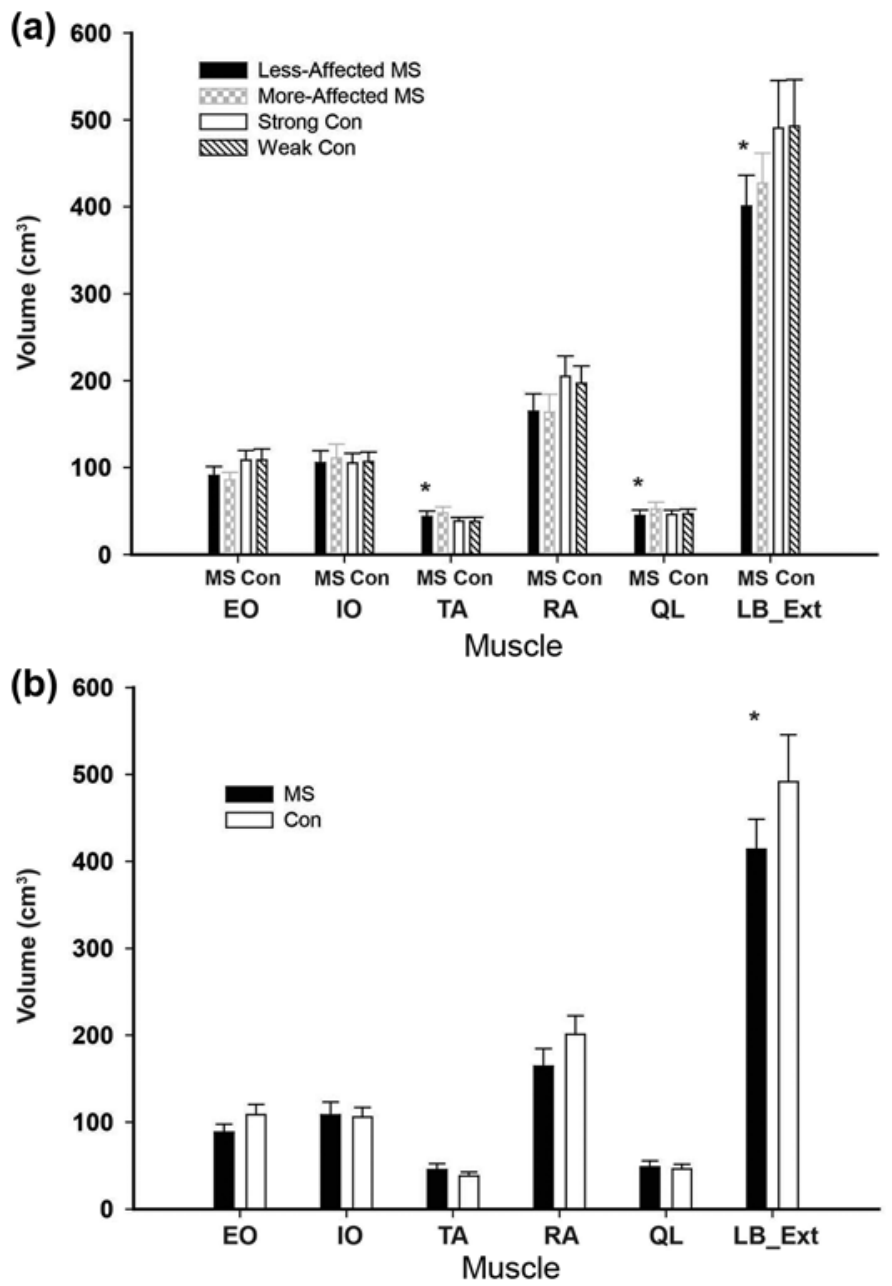

Figure 4.

(a) Mean muscle volume $\left(\mathrm{cm}^{3}\right)$ in patients with multiple sclerosis (MS) and nondisabled controls (Con) between sides of specific muscles and muscle groups. ${ }^{*} p<0.05$ between more and lessaffected sides. (b) Average muscle volume between patients with MS and nondisabled controls of specific muscles and muscle groups. ${ }^{*} p<0.05$ between MS and control. EO = external oblique, IO = internal oblique, LB Ext = low-back extensor group (multifidus, longissimus, iliocostalis), $\mathrm{QL}$ = quadratus lumborum, $\mathrm{RA}=$ rectus abdominis, $\mathrm{TA}=$ transversus abdominis.

\section{Glucose Uptake Heterogeneity}

There were no main effects or interactions for side or condition for any of the muscles $(p>0.14)$. Furthermore, within-group comparisons of side showed no differences within either group $(p>0.17)$ (Figure 5(a)).

There was no main effect of condition or muscle $x$ condition interaction $(p>0.43)$. However, a main effect of muscle was found $(p<0.01)$. Post hoc analyses
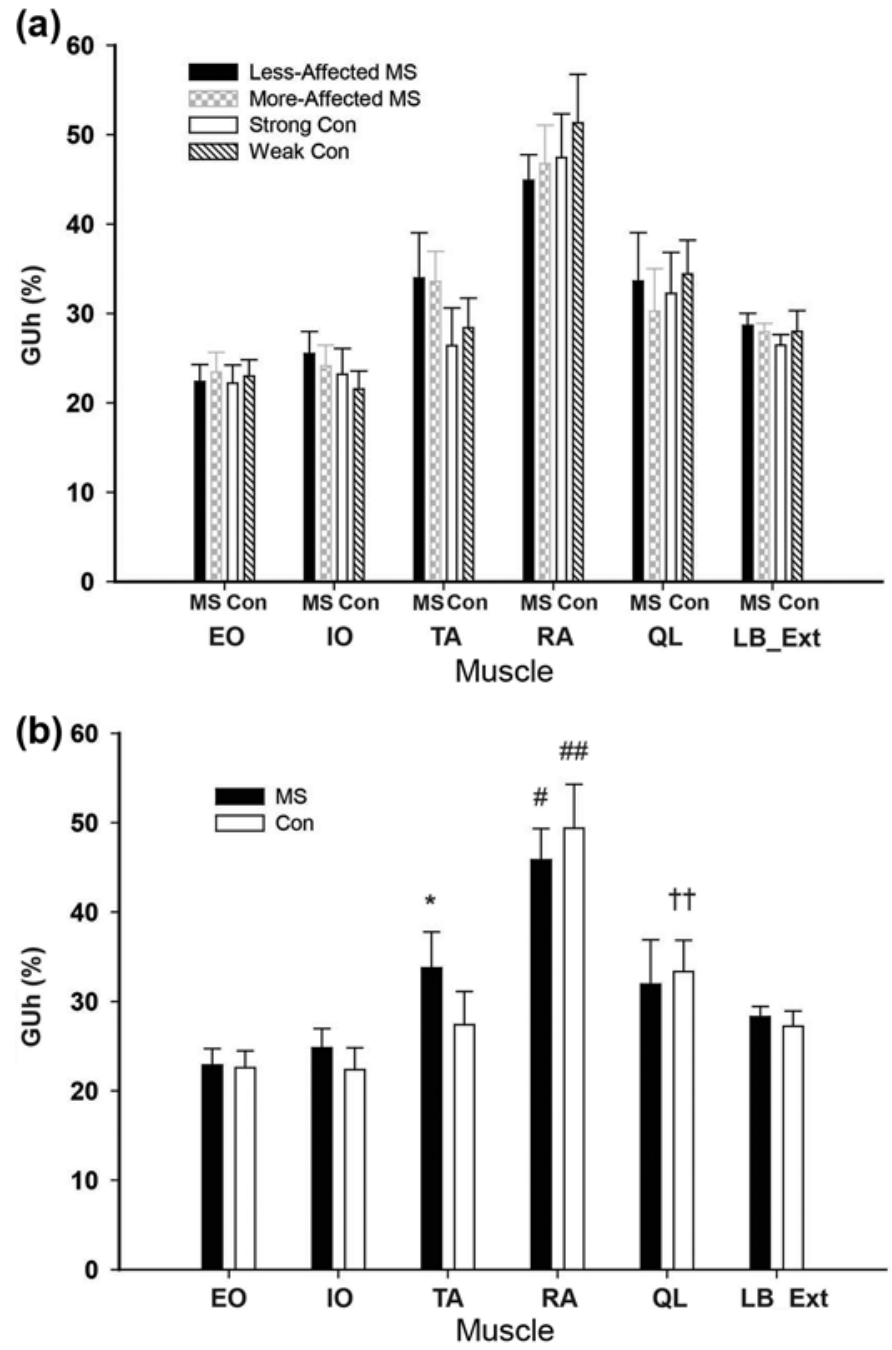

Figure 5.

(a) No difference in glucose uptake heterogeneity (GUh) in patients with multiple sclerosis (MS) or nondisabled controls (Con) between sides of specific muscles and muscle groups. (b) No difference in GUh between patients with MS and nondisabled controls of entire muscles and muscle groups. *Greater GUh than EO in patients with MS $(p<0.05) .{ }^{\#}$ Greater GUh than EO, IO, TA, QL, and LB Ext in patients with MS $(p<0.05)$.

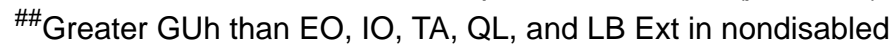
controls $(p<0.05) .{ }^{\dagger \dagger}$ Greater GUh than EO and $1 O$ in nondisabled controls $(p<0.05)$. EO $=$ external oblique, $I O=$ internal oblique, LB Ext = low-back extensor group (multifidus, longissimus, iliocostalis), $\mathrm{QL}=$ quadratus lumborum, $\mathrm{RA}=$ rectus abdominis, $\mathrm{TA}=$ transversus abdominis.

showed differences between the EO and RA/QL/TA, IO and RA/QL, LB Ext and RA, RA and TA, and QL and RA $(p<0.02)$. Within-group comparisons of muscle 
showed that the GUh of the RA was greater than the EO, IO, TA, QL, and LB Ext for both MS and controls ( $p<$ 0.01 ) (Figure 5(b)). The TA had greater GUh than the EO within the MS group $(p=0.02)$, and the GUh of the QL was greater than the EO and IO in the control group $(p<0.04)$.

\section{DISCUSSION}

This is the first study utilizing FDG-PET to quantify the activation of the core muscles in patients with MS or nondisabled individuals. The main finding was that patients with MS have imbalanced activation of the core muscles during walking as indicated by greater SUV, with the less-affected side being activated more. This finding adds to the previous results of imbalanced activation of the leg muscles and provides a more focused description of muscle activity in patients with MS during walking. This activation pattern suggests a compensatory mechanism used by patients with MS in order to maintain balance and posture during dynamic task performance such as walking.

Walking is an activity that requires bilateral activation of the legs and core muscles in order to maintain balance and posture. As a result, equal activation of both sides of the body would be expected in unaltered gait. This assumption is supported by the findings of this study, as no differences in SUV were found between sides of the nondisabled controls. However, even though there were no visible alterations in gait parameters of the mildly disabled patients with MS, side differences in activation of the core muscles were identified. Furthermore, the patients with MS walked at a slower self-selected speed than the nondisabled controls. These findings suggest that FDG-PET may be capable of identifying compensatory muscle activation strategies to maintain balance, which may influence gait parameters. Imbalanced core muscle activation could serve as an important target for rehabilitative specialists aiming to improve walking in patients with MS.

In patients with MS, volume differed between the more- and less-affected side in the individual muscles that had no difference in activity between sides (TA, QL, LB Ext), and vice versa. Furthermore, the volume was greater on the more-affected side of these muscles. This could be due to the body compensating for either a lack of muscle strength, which is associated with crosssectional area/volume, or activity (SUV). However, fur- ther research is needed to explain these findings. The greater volume may be due to an increased proportion of type 2 fibers on this side, which are known to have greater cross-sectional area. While it is suggested that muscle characteristics of patients with MS shift to a higher proportion of type 2 fibers [24,30-31], impaired neural drive to the muscles may prevent increased strength. However, it is likely that the shift in fiber type characteristics on one side of the body is not sufficient to cause imbalanced muscle volume. Furthermore, Garner and Widrick demonstrated greater atrophy in fast than in slow fibers of patients with MS [31], which would offset the expected hypertrophy that comes with a shift to type 2 fiber characteristics. The clinical relevance of the observed differences in muscle volume cannot be determined until the side differences in the strength of these muscles can be accurately assessed.

Skeletal muscle GUh is an estimation of muscle fiber activity within a muscle. Previous studies have shown altered GUh as a result of increased exercise intensity [11], disease status [32], and motor unit remodeling due to aging [10]. Our results showed no difference between the groups in GUh for any muscle, which demonstrates similar fiber recruitment between groups. These findings suggest that impaired postural stability and balance experienced by patients with MS could be caused by an inability to activate the core muscles on one side of the body rather than an altered activation strategy within the muscle. The increased GUh observed in the RA of both groups is likely due to the large aponeurosis within this muscle and the inability to distinguish between connective tissue and muscle using CT.

By implementing a novel approach to quantifying muscle activity based on FDG uptake, we were able to demonstrate for the first time the differences in core muscle activity during unassisted walking. This technique provides a unique insight into the activation patterns of the core musculature due to its ability to measure the activation of individual muscles without interference from other muscles. Measuring the activation of deep muscles, such as the IO and TA, is not feasible with either sEMG or intramuscular EMG due to limitations such as crosstalk, signal cancelation, and invasiveness of the procedure [33-34].

While we did not test core muscle strength in this study, our findings support the importance of the lateral trunk flexors during gait. Our study provides preliminary evidence that core muscle activity might be an important 
requirement of efficient walking, and thus suggests that core strength should be targeted when selecting rehabilitation-based intervention strategies aimed at improving walking capacity in patients with MS. Though in the current study we are unable to declaratively conclude how the probable compensatory activity of the lateral trunk flexors of the less affected side augments walking, we postulate that this process may be a compensatory mechanism for enhanced advancement of the more-affected limb during swing phase and/or additional whole core stabilization due to weakness of the lateral trunk flexors of the more-affected side. In people with stroke, where there is also commonly a more- and less-affected side, this concept is supported by findings of a significant association between walking ability and lateral trunk flexion strength on the side of the weaker leg [35].

Larson et al. used incremental unilateral cycling to demonstrate asymmetric peak workload and strength between legs in patients with MS [36]. Participants cycled under a ramped protocol until volitional exhaustion. The results showed that patients with MS cycled longer with the stronger leg, which implies greater fatigability in the weaker leg. We believe that these results, in combination with our findings, show that patients with MS may rely on their stronger side while performing dynamic tasks. This increased reliance on one side of the body suggests the utilization of a compensatory mechanism, which may lead to greater fatigability of the stronger side and sooner time-to-task-failure.

FDG-PET/CT may serve as an important pre-post measurement to assess the efficacy of interventional studies involving patients with MS. Using this technique would provide objective measures of interventional changes rather than the current methods of self-report or distantly related outcomes such as walking, mobility, or balance. Furthermore, previous studies have shown the challenges of diagnosing balance impairments in patients with MS [37-38]. These studies demonstrated the limitations of current methods, such as biomechanical analysis with force platforms and EMG, to identify balance impairments in patients with MS.

Despite the minimal evidence in the literature, it is commonly understood that the core is important for gait. As such, core strengthening in various forms has been the subject of intervention [16-18,39-40]. Although these studies generally have limitations in design and have used different approaches and outcomes, together they show an overall positive effect on gait and mobility out- comes. Still, it is not clear which core muscles might be the most important to target, nor the relative importance of trunk control, sway, strength, or endurance to walking. Our results provide early evidence that addressing the imbalances in core activation between the more- and less-affected sides, in particular for the lateral flexors, might be an important early target of intervention.

\section{Limitations}

The primary limitation of this study was the small sample size. While we were still able to find significant differences, other important associations might not have been detected due to lack of power. A second limitation was that walking took place on a treadmill, which is different from gait patterns and muscle activation used for overground walking; however, participants were familiarized with treadmill walking prior to beginning the test. Additionally, the lower temporal resolution of FDG-PET/ CT could be considered as a limitation. Rate of FDG uptake can provide improved temporal resolution but requires blood sampling, which was not performed in this study. Other modalities, such as EMG, are able to measure muscle activity multiple times per second. As such, it is possible that using EMG in conjunction with FDG$\mathrm{PET} / \mathrm{CT}$ may provide even greater information on skeletal muscle properties during task performance in patients with MS.

\section{Future Studies}

FDG-PET/CT is a novel technique to measure whole-muscle activity. Future studies should be conducted to investigate muscle activity in patients with MS and nondisabled controls while walking at the same speed. Additionally, the association between gait and core strength deficits and asymmetries should be further validated after properly determining the magnitude of muscle strength deficits in the core musculature. A larger sample size and varying levels of disability would provide greater insight into activation patterns and imbalances. Finally, biomechanical analysis in combination with FDG-PET/CT could help explain altered gait and balance control parameters in patients with MS. Examining FDG uptake in these situations would allow for better monitoring of disease progression and new rehabilitative strategies to be implemented. 


\section{CONCLUSIONS}

Mildly disabled patients with MS demonstrate imbalanced activation of the core muscles during walking, with the less-affected side being activated to a greater extent. Rehabilitation strategies should target the core muscles, in particular the lateral flexor group, in order to decrease the reliance on one side of the body. The lateral flexor group may be of particular importance to maintaining balance and posture while walking, and inefficient activation patterns in this group could potentially lead to higher levels of muscle fatigue.

\section{ACKNOWLEDGMENTS}

\section{Author Contributions:}

Acquisition of data: N. B. Ketelhut.

Analysis and interpretation of data: N. B. Ketelhut, J. H. Kindred, M. M. Manago, J. R. Hebert, T. Rudroff.

Drafting of manuscript: N. B. Ketelhut, J. H. Kindred, M. M. Manago, J. R. Hebert, T. Rudroff.

Critical revision of manuscript for important intellectual content:

N. B. Ketelhut, J. H. Kindred, M. M. Manago, J. R. Hebert, T. Rudroff. Obtained funding: T. Rudroff.

Financial Disclosures: The authors have declared that no competing interests exist.

Funding/Support: This material was based on work supported by the National Multiple Sclerosis Society \#PP1907 to T. Rudroff and in part by National Institutes of Health/National Center for Advancing Translational Sciences Colorado (grant UL1 TR000154). The PDDS is provided for use by the North American Research Committee on Multiple Sclerosis (NARCOMS) registry: www.narcoms.org. NARCOMS is supported in part by the Consortium of Multiple Sclerosis Centers (CMSC) and the CMSC Foundation.

Additional Contributions: The authors thank Phillip Koo, MD; Ramesh Karki, MS; and John-Michael Benson for their help in participant recruitment, image acquisition, and image analysis. We also thank Ann Hess, PhD, for her help in statistical analysis.

Institutional Review: This study and informed consent were approved by the Colorado Multiple Institutional Review Board.

Participant Follow-Up: The authors plan to inform the participants of publication of this study.

\section{REFERENCES}

1. Fox RJ, Bethoux F, Goldman MD, Cohen JA. Multiple sclerosis: Advances in understanding, diagnosing, and treating the underlying disease. Cleve Clin J Med. 2006; 73(1):91-102. [PMID:16444920]

2. Hoang PD, Gandevia SC, Herbert RD. Prevalence of joint contractures and muscle weakness in people with multiple sclerosis. Disabil Rehabil. 2014;36(19):1588-93.

[PMID:24236496]

http://dx.doi.org/10.3109/09638288.2013.854841

3. Thoumie P, Lamotte D, Cantalloube S, Faucher M, Amarenco G. Motor determinants of gait in 100 ambulatory patients with multiple sclerosis. Mult Scler. 2005;11(4): 485-91. [PMID: 16042234]

http://dx.doi.org/10.1191/1352458505ms1176oa

4. Rudroff T, Kindred JH, Koo PF, Karki R, Hebert JR. Asymmetric glucose uptake in leg muscles of patients with multiple sclerosis during walking detected by $\left[{ }^{18} \mathrm{~F}\right]-\mathrm{FDG}$ PET/CT. NeuroRehabilitation. 2014;35(4):813-23.

[PMID:25323085]

5. Heesen C, Böhm J, Reich C, Kasper J, Goebel M, Gold SM. Patient perception of bodily functions in multiple sclerosis: Gait and visual function are the most valuable. Mult Scler. 2008;14(7):988-91. [PMID:18505775] http://dx.doi.org/10.1177/1352458508088916

6. Chung LH, Remelius JG, Van Emmerik RE, Kent-Braun JA. Leg power asymmetry and postural control in women with multiple sclerosis. Med Sci Sports Exerc. 2008; 40(10):1717-24. [PMID:18799980] http://dx.doi.org/10.1249/MSS.0b013e31817e32a3

7. Lanzetta D, Cattaneo D, Pellegatta D, Cardini R. Trunk control in unstable sitting posture during functional activities in healthy subjects and patients with multiple sclerosis. Arch Phys Med Rehabil. 2004;85(2):279-83.

[PMID:14966714]

http://dx.doi.org/10.1016/j.apmr.2003.05.004

8. Pappas GP, Olcott EW, Drace JE. Imaging of skeletal muscle function using (18)FDG PET: Force production, activation, and metabolism. J Appl Physiol. 2001;90(1):329-37. [PMID:11133926]

9. Rudroff T, Kalliokoski KK, Block DE, Gould JR, Klingensmith WC 3rd, Enoka RM. PET/CT imaging of age- and task-associated differences in muscle activity during fatiguing contractions. J Appl Physiol. 2013;114(9):1211-19.

[PMID:23412899]

http://dx.doi.org/10.1152/japplphysiol.01439.2012

10. Rudroff T, Kindred JH, Benson JM, Tracy BL, Kalliokoski KK. Greater glucose uptake heterogeneity in knee muscles of old compared to young men during isometric contractions detected by [(18)F]-FDG PET/CT. Front Physiol. 2014;5:198. [PMID:24904432] http://dx.doi.org/10.3389/fphys.2014.00198

11. Heinonen I, Nesterov SV, Kemppainen J, Fujimoto T, Knuuti J, Kalliokoski KK. Increasing exercise intensity reduces heterogeneity of glucose uptake in human skeletal muscles. PLoS ONE. 2012;7(12):e52191. [PMID:23284929] http://dx.doi.org/10.1371/journal.pone.0052191 
12. Kindred JH, Ketelhut NB, Rudroff T. Glucose uptake heterogeneity of the leg muscles is similar between patients with multiple sclerosis and healthy controls during walking. Clin Biomech (Bristol, Avon). 2015;30(2):159-65. [PMID:25541392]

13. Piepers I, Boudt P, Van Tongel A, De Wilde L. Evaluation of the muscle volumes of the transverse rotator cuff force couple in nonpathologic shoulders. J Shoulder Elbow Surg. 2014;23(7):e158-62. [PMID:24342371] http://dx.doi.org/10.1016/j.jse.2013.09.027

14. White SG, McNair PJ. Abdominal and erector spinae muscle activity during gait: The use of cluster analysis to identify patterns of activity. Clin Biomech (Bristol, Avon). 2002;17(3):177-84. [PMID:11937255] http://dx.doi.org/10.1016/S0268-0033(02)00007-4

15. Swinnen E, Baeyens JP, Pintens S, Van Nieuwenhoven J, Ilsbroukx S, Clijsen R, Buyl R, Goossens M, Meeusen R, Kerckhofs E. Trunk muscle activity during walking in persons with multiple sclerosis: The influence of body weight support. NeuroRehabilitation. 2014;34(2):323-35. [PMID:24419023]

16. Freeman JA, Gear M, Pauli A, Cowan P, Finnigan C, Hunter H, Mobberley C, Nock A, Sims R, Thain J. The effect of core stability training on balance and mobility in ambulant individuals with multiple sclerosis: A multicentre series of single case studies. Mult Scler. 2010; 16(11):1377-84. [PMID:20699285] http://dx.doi.org/10.1177/1352458510378126

17. Guclu-Gunduz A, Citaker S, Irkec C, Nazliel B, BaturCaglayan HZ. The effects of pilates on balance, mobility and strength in patients with multiple sclerosis. NeuroRehabilitation. 2014;34(2):337-42. [PMID:23949064]

18. Keser I, Kirdi N, Meric A, Kurne AT, Karabudak R. Comparing routine neurorehabilitation program with trunk exercises based on Bobath concept in multiple sclerosis: Pilot study. J Rehabil Res Dev. 2013;50(1):133-40.

[PMID:23516089]

http://dx.doi.org/10.1682/JRRD.2011.12.0231

19. Hohol MJ, Orav EJ, Weiner HL. Disease steps in multiple sclerosis: A simple approach to evaluate disease progression. Neurology. 1995;45(2):251-55. [PMID:7854521] http://dx.doi.org/10.1212/WNL.45.2.251

20. Hohol MJ, Orav EJ, Weiner HL. Disease steps in multiple sclerosis: A longitudinal study comparing disease steps and EDSS to evaluate disease progression. Mult Scler. 1999; 5(5):349-54. [PMID:10516779] http://dx.doi.org/10.1177/135245859900500508

21. Marrie RA, Goldman M. Validity of performance scales for disability assessment in multiple sclerosis. Mult Scler. 2007;13(9):1176-82. [PMID:17623733] http://dx.doi.org/10.1177/1352458507078388
22. Learmonth YC, Motl RW, Sandroff BM, Pula JH, Cadavid D. Validation of Patient Determined Disease Steps (PDDS) scale scores in persons with multiple sclerosis. BMC Neurol. 2013;13:37. [PMID:23617555]

http://dx.doi.org/10.1186/1471-2377-13-37

23. Kobelt G, Berg J, Atherly D, Hadjimichael O. Costs and quality of life in multiple sclerosis: A cross-sectional study in the United States. Neurology. 2006;66(11):1696-1702. [PMID:16769943] http://dx.doi.org/10.1212/01.wnl.0000218309.01322.5c

24. Kent-Braun JA, Ng AV, Castro M, Weiner MW, Gelinas D, Dudley GA, Miller RG. Strength, skeletal muscle composition, and enzyme activity in multiple sclerosis. J Appl Physiol. 1997;83(6):1998-2004. [PMID:9390973]

25. Andreasen AK, Jakobsen J, Petersen T, Andersen $H$. Fatigued patients with multiple sclerosis have impaired central muscle activation. Mult Scler. 2009;15(7):818-27. [PMID:19465444] http://dx.doi.org/10.1177/1352458509105383

26. Lambert CP, Archer RL, Evans WJ. Muscle strength and fatigue during isokinetic exercise in individuals with multiple sclerosis. Med Sci Sports Exerc. 2001;33(10):1613-19. [PMID:11581542] http://dx.doi.org/10.1097/00005768-200110000-00001

27. Glenn JM, Galey M, Edwards A, Rickert B, Washington TA. Validity and reliability of the abdominal test and evaluation systems tool (ABEST) to accurately measure abdominal force. J Sci Med Sport. 2015;18(4):457-62.

[PMID:25024133]

28. Goodpaster BH, Kelley DE, Thaete FL, He J, Ross R. Skeletal muscle attenuation determined by computed tomography is associated with skeletal muscle lipid content. J Appl Physiol. 2000;89(1):104-10. [PMID:10904041]

29. Martin L, Birdsell L, Macdonald N, Reiman T, Clandinin MT, McCargar LJ, Murphy R, Ghosh S, Sawyer MB, Baracos VE. Cancer cachexia in the age of obesity: Skeletal muscle depletion is a powerful prognostic factor, independent of body mass index. J Clin Oncol. 2013;31(12):1539-47. [PMID:23530101] http://dx.doi.org/10.1200/JCO.2012.45.2722

30. Lenman AJ, Tulley FM, Vrbova G, Dimitrijevic MR, Towle JA. Muscle fatigue in some neurological disorders. Muscle Nerve. 1989;12(11):938-42. [PMID:2608089] http://dx.doi.org/10.1002/mus.880121111

31. Garner DJ, Widrick JJ. Cross-bridge mechanisms of muscle weakness in multiple sclerosis. Muscle Nerve. 2003; 27(4):456-64. [PMID:12661047] http://dx.doi.org/10.1002/mus.10346

32. Watanabe K, Miyamoto T, Tanaka Y, Fukuda K, Moritani T. Type 2 diabetes mellitus patients manifest characteristic spatial EMG potential distribution pattern during sustained isometric contraction. Diabetes Res Clin Pract. 2012;97(3): 
468-73. [PMID:22483576]

http://dx.doi.org/10.1016/j.diabres.2012.03.004

33. Yang KH, Park DJ. Reliability of ultrasound in combination with surface electromyogram for evaluating the activity of abdominal muscles in individuals with and without low back pain. J Exerc Rehabil. 2014;10(4):230-35. [PMID:25210698] http://dx.doi.org/10.12965/jer.140138

34. Kim CY, Choi JD, Kim SY, Oh DW, Kim JK, Park JW. Comparison between muscle activation measured by electromyography and muscle thickness measured using ultrasonography for effective muscle assessment. J Electromyogr Kinesiol. 2014;24(5):614-20. [PMID:25088820] http://dx.doi.org/10.1016/j.jelekin.2014.07.002

35. Bohannon RW. Lateral trunk flexion strength: Impairment, measurement reliability and implications following unilateral brain lesion. Int J Rehabil Res. 1992;15(3):249-51. [PMID:1428392] http://dx.doi.org/10.1097/00004356-199209000-00010

36. Larson RD, McCully KK, Larson DJ, Pryor WM, White LJ. Bilateral differences in lower-limb performance in individuals with multiple sclerosis. J Rehabil Res Dev. 2013; 50(2):215-22. [PMID:23761002] http://dx.doi.org/10.1682/JRRD.2011.10.0189

37. Cameron MH, Horak FB, Herndon RR, Bourdette D. Imbalance in multiple sclerosis: A result of slowed spinal somatosensory conduction. Somatosens Mot Res. 2008; 25(2):113-22. [PMID:18570015]

http://dx.doi.org/10.1080/08990220802131127

38. Corradini ML, Fioretti S, Leo T, Piperno R. Early recognition of postural disorders in multiple sclerosis through movement analysis: A modeling study. IEEE Trans Biomed Eng. 1997;44(11):1029-38. [PMID:9353982]

http://dx.doi.org/10.1109/10.641330

39. Freeman J, Fox E, Gear M, Hough A. Pilates based core stability training in ambulant individuals with multiple sclerosis: A protocol for a multi-centre randomized controlled trial. BMC Neurol. 2012;12:19. [PMID:22289169]

40. Smedal T, Lygren H, Myhr KM, Moe-Nilssen R, Gjelsvik B, Gjelsvik O, Strand LI. Balance and gait improved in patients with MS after physiotherapy based on the Bobath concept. Physiother Res Int. 2006;11(2):104-16. Erratum in Physiother Res Int. 2007;12(1):52.

[PMID:16808091]

http://dx.doi.org/10.1002/pri.327

Submitted for publication January 10, 2015. Accepted in revised form April 29, 2015.

This article and any supplementary material should be cited as follows:

Ketelhut NB, Kindred JH, Manago MM, Hebert JR, Rudroff T. Core muscle characteristics during walking of patients with multiple sclerosis. J Rehabil Res Dev. 2015; 52(6): 713-24.

http://dx.doi.org/10.1682/JRRD.2015.01.0006

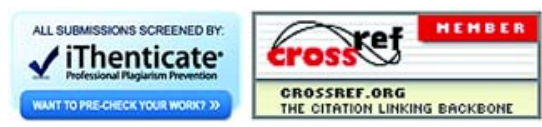


